\title{
Editorial
}

\section{Importance of Awareness of Adverse Drug Reaction Reporting During the COVID-19 Pandemic}

Heather Ellis, PharmD, ${ }^{1}$ Hale Z. Toklu, PhD, MPharm²

\section{Abstract}

Author affiliations are listed at the end of this article.

Correspondence to: Heather Ellis, PharmD (Heather.Ellis88@gmail. com)

\section{Description}

Health care professionals hold the responsibility of reporting any adverse drug reactions in order to learn about new therapy and how best to safely care for our patients. The information derived from case publications and FDA MedWatch reports are essential to accumulate information and increase awareness for the possible risks of new drugs.

\section{Keywords}

SARS-CoV-2; COVID-19; coronavirus Infections; drug-related side effects and adverse reactions; medication; drug safety; adverse reaction; pharmacovigilance

What is certain is that we are uncertain. During this unprecedented time of the COVID-19 pandemic, treatment modalities, transmission best practices and care for COVID-19 patients have fluctuated frequently. In a time where the answer is not readily available, we are required to uphold our professional obligation to ensure we learn and practice based on all available information.

Since the start of the pandemic in early 2020 , the world of healthcare has seen change unlike any other in these modern times. Treatment guidance has changed drastically, sometimes day to day. The reason behind this is, as we learn more, we are able to shift decision-making to the new best practices to care for patients with a virus that was not previously known.

In the beginning of the pandemic access to one of the treatment options, an anti-viral agent called remdesivir was limited. Eventually there was an Emergency Use Authorization (EUA) for the therapy, as it did not hold a current approved indication for COVID-19.' In knowing this medication had not been studied for this indication and subsequently did not yet have approval for it, health care providers had a greater responsibility to keep a close eye on therapy.

One of the ways to monitor this particular new therapy as health care providers is through reports of unexpected or unwanted reactions to the medication. This is known as an adverse drug reaction. Since remdesivir has not been previously recommended or administered for this indication-reporting of any adverse reactions and learning as we go becomes imperative. This therapy is being used across the globe and there are processes in place for these reports to be shared to understand more about possible risks or undesired outcomes.

Within each facility in a healthcare system, there is a process for reporting adverse drug reactions. The issue then becomes how this information is shared with other facilities and healthcare systems caring for the same types of patients with this therapy. The U.S. Food and Drug Administration (FDA) MedWatch is a reporting program for adverse events. ${ }^{2}$ Health professionals and patients can submit reports. This reporting is voluntary and can help the FDA identify previously unknown risks for med-

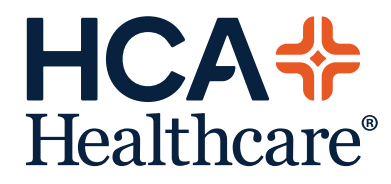

www.hcahealthcarejournal.com

(C) 2020 HCA Physician Services, Inc. d/b/a Emerald Medical Education
HCA Healthcare Journal of Medicine 
ications or medical products.

You may think that the reporting of these adverse drug reactions would consistently occur. In reality, reporting of adverse reactions are not always common. Facilities operating under a "Just Culture" find themselves in the best position to increase reporting. A "Just Culture" is the mentality that there is support in an organization for reporting of issues and no punitive action toward the reporter.

The Institute of Safe Medication Practices (ISMP) is a great resource for patient safety and best practices to ensure safe use of medicine. In organizations known to utilize support from ISMP best practices, a "Just Culture" is common and there is a healthy relationship with reporting adverse drug reactions. We can see how the reporting of reactions is necessary and that when reports are accumulated and monitored for trends reporting can help shape practice and care of patients.

ISMP recently published an acute care medication safety alert newsletter with the main article focusing on reported medication errors with remdesivir use under the EUA. ${ }^{3}$ Errors with wrong formulation, wrong preparation and incorrect storage have all been reported. ${ }^{3}$ Recently, the final report for remdesivir was published, ${ }^{4}$ and remdesivir was approved by the FDA for treatment of adult and pediatric patients over 12 years of age and weighing at least 40 kilograms for the treatment of COVID-19 requiring hospitalization. ${ }^{4}$ As part of the EUA, which still exists after the FDA approval for certain indications, adverse drug reaction reporting (including errors) to FDA MedWatch is required of the health care provider within 7 days of the event.

As mentioned in the beginning of this article, dramatic changes in therapy for treatment of COVID-19 continues. Remdesivir therapy has already shifted in terms of options for treatment of the infection. Recently, in November 2020, baricitinib was approved under an EUA to be used in combination with remdesivir for the treatment of COVID-19 in hospitalized adult and pediatric patients requiring supplemental oxygen, invasive mechanical ventilation or extracorporeal membrane oxygenation (ECMO). ${ }^{5}$ Two new medications have now been approved under EUAs. ${ }^{6,7}$ Also in November, bamlanivimab was approved for the treatment of mild to moderate COVID-19 in adult and pediatric patients. ${ }^{6}$ It is important to note, bamlanivimab is not approved for hospitalized patients. ${ }^{6}$ The other therapy recently given an EUA in November is casirivimab and imdevimab, which must be administered together for the treatment of COVID-19 in adults and pediatric patients and also is not authorized for hospitalized patients. ${ }^{\text {? }}$

With treatment options growing, the need for reporting of any adverse reactions does not diminish but increases. Treatment of COVID-19 in many different settings only furthers the need for reporting, as the reactions may not be witnessed by the same health care providers in the inpatient and outpatient settings. In some instances in the outpatient setting, patients should be educated to submit a report to FDA MedWatch. ${ }^{1}$

As health care professionals who may prepare, dispense and/or administer and monitor patients receiving these new agents, we hold the responsibility of reporting any adverse drug reactions to continue to learn about these therapies and how best to safely care for our patients. ${ }^{8}$ Reporting should follow local internal reporting system process as well as FDA MedWatch. Especially amidst pandemic, every report is valuable. Case studies are compiled with new information, including adverse reactions, on patients treated with remdesivir and other recent EAU therapies. All of this information is needed to continue treatment forward and hold patient safety in the highest regard while fighting against the pandemic.

\section{Conflicts of Interest}

The authors declare they have no conflicts of interest.

Dr. Ellis is an employee of North Florida Regional Medical Center, a hospital affiliated with the journal's publisher.

This research was supported (in whole or in part) by HCA Healthcare and/or an HCA Healthcare affiliated entity. The views expressed in this publication represent those of the author(s) and do not necessarily represent the official views of HCA Healthcare or any of its affiliated entities. 
Ellis and Toklu. (2020) 1:6. https://doi.org/10.36518/2689-0216.1206

\section{Author Affiliations}

1. North Florida Regional Medical Center, Medication Safety Advanced Clinical

Pharmacist, Gainesville, FL

2. University of Central Florida College of Medicine, Department of Clinical Sciences, Gainesville, FL

\section{References}

1. US Food and Drug Administration. FDA Approves First Treatment for COVID-19. October 22, 2020. https://www.fda.gov/news-events/ press-announcements/fda-approves-first-treatment-covid-19. Accessed November 05, 2020.

2. MedWatch: The FDA Safety Information and Adverse Event Reporting Program. https:// www.fda.gov/safety/medwatch-fda-safety-information-and-adverse-event-reporting-program. Accessed December 11, 2020.

3. FDA Advise-ERR: Reported Medication Errors with Veklury (Remdesivir) Emergency Use Authorization. Acute Care ISMP Medication Safety Alert!. 2020 September;25(18). https:// www.ismp.org/resources/fda-advise-err-reported-medication-errors-veklury-remdesivir-emergency-use-authorization

4. Beigel JH, Tomashek KM, Dodd LE, et al. Remdesivir for the Treatment of Covid-19 - Final Report. N Engl J Med. 2020;383(19):1813-1826. https://doi.org/10.1056/nejmoa2007764

5. US Food and Drug Administration. Coronavirus (COVID-19) Update: FDA Authorizes Drug Combination for Treatment of COVID-19. November 19, 2020. https://www.fda.gov/news-events/ press-announcements/coronavirus-covid-19-update-fda-authorizes-drug-combination-treatment-covid-19. Accessed December 11, 2020.

6. US Food and Drug Administration. Coronavirus (COVID-19) Update: FDA Authorizes Monoclonal Antibodies for Treatment of COVID-19. November 21, 2020. https://www.fda.gov/ news-events/press-announcements/coronavirus-covid-19-update-fda-authorizes-monoclonal-antibodies-treatment-covid-19. Accessed December 11, 2020.

7. US Food and Drug Administration. Coronavirus (COVID-19) Update: FDA Authorizes Monoclonal Antibody for Treatment of COVID-19. November 9, 2020. https://www.fda.gov/news-events/ press-announcements/coronavirus-covid-19-update-fda-authorizes-monoclonal-antibody-treatment-covid-19. Accessed December 11, 2020.

8. Toklu HZ, Mensah E. Why do we need pharmacists in pharmacovigilance systems? Online J Public Health Inform. 2016 Sep 15;8(2):e193. https://doi.org/10.5210/ojphi.v8i2.6802 